No hacer: de las recomendaciones a la acción

\title{
Do not do: from recommendations to action
}

https://doi.org/10.23938/ASSN.0380

C. Bermúdez Tamayo ${ }^{1,2,3}$, A. Olry de Labry Lima ${ }^{1,2,3}$, L. García Mochón ${ }^{1,2,3}$

\section{Sr. Editor:}

Los autores agradecen los comentarios al artículo "Priorización de actividades clínicas no recomendadas en Atención Primaria" ${ }^{1}$ realizados por Mira y $\mathrm{col}^{2}$, quienes recuerdan que han pasado ya diez años desde las primeras iniciativas internacionales dirigidas a identificar las tecnologías o procedimientos de dudoso valor, y cinco desde el inicio del proyecto Compromiso por la Calidad de las Sociedades Científicas liderado por el Ministerio de Sanidad y Consumo de nuestro país.

Los autores describen en su carta una serie de proyectos e iniciativas que se están llevando a cabo en España y que, sin duda, van a ampliar el cuerpo del conocimiento en esta área, tan necesario para avanzar. Dos hechos ponen en evidencia que hay que seguir trabajando e investigando: en primer lugar, el uso de estas tecnologías sigue siendo frecuente $\mathrm{y}$, en algunos casos, sigue aumentando ${ }^{2} \mathrm{y}$, en segundo lugar, la constatación de que la difusión de las listas de "no hacer" en España es bastante mejo- rable, ya que la iniciativa es desconocida, al menos, para el 58\% de los médicos hospitalarios y para el $56 \%$ de los profesionales de AP (según datos de los mismos autores) ${ }^{3}$.

Según estudios recientes ${ }^{4}$, los profesionales sanitarios reconocen que incluso cuando la evidencia científica se disemina en forma de documentos de consenso, como es el caso, hay muchos que no cambiarán sus prácticas. Incluso se ha constatado que, aunque los profesionales manifiesten que aceptan y tienen la intención de usar la evidencia, la aplicación efectiva tiende a disminuir, desviarse del uso pretendido o adoptar nuevas formas. Esto sugiere que la implementación exitosa de la desadopción es más compleja de lo que se podría pensar, apuntando a múltiples niveles de actuación ${ }^{5}$.

Por todo ello, los autores creen que una vez que haya evidencia y consenso sobre las tecnologías innecesarias, llega el momento de pasar a la acción. Así, parece plausible pensar en implementar aquellas intervenciones, para la desadopción de tecnologías sanitarias, que se muestren coste-
1. Escuela Andaluza de Salud Pública (EASP). Campus Universitario de Cartuja. Granada.

2. Instituto de Investigación Biosanitaria IBS. Granada. Hospitales Universitarios de Granada/Universidad de Granada. Granada.

3. CIBER en Epidemiología y Salud Pública (CIBERESP). España.

\author{
Correspondencia: \\ Clara Bermúdez Tamayo \\ Escuela Andaluza de Salud Pública \\ Campus Universitario de Cartuja \\ Cuesta del Observatorio, 4 \\ 18011 Granada \\ E-mail: clara.bermudez.easp@juntadeandalucia.es
}


efectivas ya que, a su vez, aumentarán la seguridad del paciente, disminuirán costes, mejorarán la calidad asistencial y probablemente la satisfacción del paciente.

Existe un incipiente cuerpo de conocimiento que muestra experiencias y resultados sobre intervenciones en la desadopción de tecnologías sanitarias ${ }^{5}$. Colla y col ${ }^{6}$ destacan en su revisión sistemática que aquellas intervenciones que utilizaban estrategias multifacéticas y que consideran las barreras y los agentes facilitadores (cultura, características del sistema sanitario, actitudes de los profesionales y la presión que ejerce la industria o los pacientes, etc. ${ }^{4}$ ) han mostrado un mayor potencial e impacto ${ }^{7,8}$. Algunos ejemplos de estas estrategias serían la formación directa o por pares al paciente, los clinical pathways, el apoyo del equipo clínico, la formación de los clínicos (multicomponente con apoyo clínico continuo,) o la auditoría y feedback ${ }^{9}$. Adicionalmente se deben considerar múltiples actores creando sinergias entre individuos, equipos, organizaciones y entornos, lo que requiere desarrollar colaboraciones entre niveles (primaria y hospital), profesionales (responsables de la toma de decisiones/gestores y profesionales sanitarios), así como asociaciones de pacientes y ciudadanos ${ }^{10,11}$.

Realmente, trabajar en esta línea es un verdadero reto. Pocos estudios han explorado los desafíos conceptuales y operativos inherentes a la reducción o eliminación de una práctica que se ha considerado inefectiva, inapropiada o perjudicial. Para ello, es necesaria una comprensión más profunda de los factores que afectan al cambio de comportamiento de los individuos en las organizaciones. En este sentido, la toma de decisiones clínicas, como las decisiones humanas, parece ser el resultado de dos modos distintos de procesamiento cognitivo $^{12}$ : sistema tipo 2 , el proceso consciente de evaluar opciones basadas en una combinación de utilidad, riesgo, capacidades y/o influencias sociales, y la cognición automática o sistema tipo 1 , un conjunto de procesos en gran parte inconscientes que ocurren en respuesta a señales ambientales o emotivas y se basan en heurísticos arraigados, previamente aprendidos.
Empiezan a presentarse modelos basados en teorías centradas en la comprensión de cómo los profesionales toman decisiones y se comportan a la hora de gestionar procedimientos de bajo valor ${ }^{8}$. Scott y col $^{13}$ revisan los sesgos cognitivos más comunes que influyen en la toma de decisiones en la práctica clínica y describen estrategias metacognitivas para mitigarlos. Sin embargo, muy pocos estudios han evaluado estas estrategias de desadopción para probar su efectividad. Se abre, por tanto, un ámbito en el cual habrá que aportar evidencia que ayude a pasar de las recomendaciones a la acción.

\section{BIBLIOGRAFÍA}

1. García-Mochón L, Olry de Labry A, BermúdezTAmayo L. Priorización de actividades clínicas no recomendadas en Atención Primaria. An Sist Sanit Navar 2017; 40: 401-412. https:// doi.org/10.23938/ASSN.0380

2. Mira JJ, Agra Y, Astier P, Caro J, Silvestre C, OliVERA $G$ et al. Dejar de hacer lo que no hay que hacer. An Sist Sanit Navar 2019; 42: 101-103. https://doi.org/10.23938/ASSN.0371

3. Mira JJ, Carrillo I, Pérez-Pérez P, Olivera G, Silvestre C, Nebot C et al. Grado de conocimiento entre médicos de familia, pediatras y enfermería de la campaña Compromiso por la Calidad y de recomendaciones No hacer para Atención Primaria. An Sist Sanit Navar 2018; 41: 47-55. https://doi.org/10.23938/ ASSN.0228

4. Scott IA, Elshaug AG. Foregoing low-value care: how much evidence is needed to change beliefs? Intern Med J 2013; 43: 107-109. https://doi.org/10.1111/imj.12065

5. Pathirana T, Clark J, Moynihan R. Mapping the drivers of overdiagnosis to potential solutions. BMJ 2017; 358: j3879. https://doi. org/10.1136/bmj.j3879

6. Colla CH, Mainor AJ, Hargreaves C, Sequist T, MoRDEN N. Interventions aimed at reducing use of low-value health services: a systematic review. Med Care Res Rev 2017; 74: 507-550. https://doi.org/10.1177/1077558716656970

7. Colquhoun HL, Squires JE, Kolehmainen N, Fraser C, GRIMShaW JM. Methods for designing interventions to change healthcare professionals behaviour: a systematic review. Implement Sci 2017; 12: 30. https://doi.org/10.1186/ s13012-017-0560-5 
8. Helfrich CD, Rose AJ, Hartmann CW, van BodeGom-Vos L, Graham ID, Wood SJ et al. How the dual process model of human cognition can inform efforts to de-implement ineffective and harmful clinical practices: A preliminary model of unlearning and substitution. J Eval Clin Pract 2018; 24: 198-205. https://doi. org/10.1111/jep. 12855

9. Ivers N, Jamtvedt G, Flottorp S, Young JM, ODGaARD-Jensen J, French SD. Audit and feedback: effects on professional practice and healthcare outcomes. Cochrane Database Syst Rev 2012; 6.

10. Niven DJ, Mrklas KJ, Holodinsky JK, Straus SE, HeMmelgaRn BR, JeFFs LP et al. Towards understanding the de-adoption of low-value clinical practices: a scoping review. BMC Med 2015; 13 : 255. https://doi.org/10.1186/s12916-015-0488.
11. Harris C, Ko H, Waller C, Sloss P, Williams P. Sustainability in health care by allocating resources effectively (SHARE) 4: exploring opportunities and methods for consumer engagement in resource allocation in a local healthcare setting. BMC Health Serv Res 2017; 17: 329. https://doi.org/10.1186/s12913017-2212-5

12. KAHNEMAN D. Maps of bounded rationality: psychology for behavioral economics. Am Econ Rev 2003; 93: 1449-1475. https://doi. org/10.1257/000282803322655392

13. Scott IA, Soon J, Elshaug AG, Lindner R. Countering cognitive biases in minimising low value care. Med J Aust 2017; 206: 407-411. https://doi.org/10.5694/mja16.00999 
